Article

\title{
Investigation of Similarity in the Spectra between Short- and Long-Duration Gamma-ray Bursts
}

\author{
Takanori Sakamoto* (D), Yuuki Yoshida and Motoko Serino \\ Department of Physics andMathematics, College of Science and Engineering, Aoyama Gakuin University, \\ 5-10-1 Fuchinobe, Chuo-ku, Sagamihara-shi, Kanagawa 252-5258, Japan; yusakamoto.lab.0927@gmail.com (Y.Y.); \\ serino@phys.aoyama.ac.jp (M.S.) \\ * Correspondence: tsakamoto@phys.aoyama.ac.jp; Tel.: +81-42-759-6275
}

Received: 8 August 2018; Accepted: 26 September 2018; Published: 3 October 2018

\begin{abstract}
We investigated the spectral properties of the prompt emission for short- and long-duration gamma-ray bursts (GRBs) using the Fermi Gamma-ray Burst Monitor data. In particular, we focused on comparing the spectral properties of short GRBs and the initial $2 \mathrm{~s}$ of long GRBs, motivated by the previous study of Ghirlanda et al. (2009). We confirmed the similarity in the low energy photon index $\alpha$ between short GRBs and the initial $2 \mathrm{~s}$ of long GRBs. Since about a quarter of our spectra of both short GRBs and the initial $2 \mathrm{~s}$ of long GRBs show $\alpha$ to be shallower than $-2 / 3$, it is difficult to understand in the context standard synchrotron emission.
\end{abstract}

Keywords: gamma-ray burst; prompt emission; spectrum

\section{Introduction}

The origin of short-duration gamma-ray bursts (hereafter short GRBs) is receiving great attention in the field of astrophysics. During the second Laser Interferometer Gravitational wave Observatory and Virgo observation run in 2017, the first gravitational wave event from the merging neutron stars, GW 170817, was observed [1]. The Fermi Gamma-ray Space Telescope (Fermi) Gamma-ray Burst Monitor (GBM) and the Anti-Coincidence Shield (ACS) of the SPI spectrometer on board the INTernational Gamma-ray Astrophysics Laboratory (INTEGRAL) detected a possible short GRB 170817A about $1.7 \mathrm{~s}$ after the merger time [2]. GRB 170817A has the $t_{90}$ duration of $2.0 \pm 0.5 \mathrm{~s}$ and the total radiation energy was 3-4 orders of magnitude lower than that of the typical short GRBs [3]. The origin of the weakness of GRB 170817A is still unclear. However, the recent X-ray e.g., [4,5] and radio e.g., [6] observations at the late phase suggest that the weak emission is consistent with an off-axis viewing effect (a weakened relativistic beaming effect; $[7,8]$ ) of a typical short GRB jet.

One of the well-known properties of short GRBs is the hardness of their spectra in the prompt emission. A short GRB tends to have a harder spectrum than a long duration GRB (hereafter long GRB) e.g., [9]. Ghirlanda et al. [10] show that the hardness of short GRBs is driven by a shallower (harder) low energy photon index $\alpha$ compared to that of long GRBs rather than the peak energy in the $v \mathrm{~F}_{v}$ spectrum $E_{\text {peak }}$ by the Compton Gamma-ray Observatory Burst And Transient Source Experiment (BATSE) sample. Further investigation with the much larger GRB sample confirmed this spectral difference between short GRBs and long GRBs [11]. Ghirlanda et al. [11] also pointed out that a spectrum of the initial part (the first 1-2 $\mathrm{s}$ of the emission) of a long GRB shows a similarity to a short GRB. Both $\alpha$ and $E_{\text {peak }}$ of short GRBs and the initial part of long GRBs have statistically similar distributions. This result indicates that a similar radiation process is involved between short and long GRBs.

The spectral evolution between long GRBs and short GRBs shows a similar behavior. Hakkila \& Preece [12] resolved individual pulses during the prompt emission, and demonstrated the similarities of the pulse characteristics between long GRBs and short GRBs. They also suggested that the general 
spectral evolution seen over the burst episode can be understood by the hard-to-soft evolution of the individual pulse. According to Ghirlanda et al. [13], $E_{\text {peak }}$ of time-resolved spectra of both long and short GRBs shows a positive correlation to the peak fluxes (or luminosity). Those previous works shed light on the similarity in the radiation process between long and short GRBs despite the different progenitors.

In this paper, we report the comparison of the spectral properties of a prompt emission between short and long GRBs by Fermi GBM data. The Fermi GBM possesses a large GRB sample with a good spectral coverage to derive the spectral parameters of a prompt emission for both short and long GRBs. It is worth investigating the similarity in the spectral properties between short and long GRBs reported by the BATSE sample using data of a different GRB instrument. In Section 2, we describe the investigation of a possible systematic error in the Fermi GBM data, the analysis method and the sample selection. We report the result of the comparisons of the prompt spectral parameters among short GRBs, long GRBs and the initial $2 \mathrm{~s}$ of long GRBs in Section 3. We discuss and summarize our results in Section 4. The quoted errors are at the $90 \%$ confidence level.

\section{Analysis}

The HEASOFT version 6.21 and the Fermi Science tool version v10r0p5 are used throughout the analysis. The spectrum of the Neil Gehrels Swift Observatory [14] Burst Alert Telescope [15] data is generated following the BAT analysis thread. ${ }^{1}$ The spectrum of Fermi GBM data is extracted from the Time-Tagged Event (TTE) data [16] using gtbin. The background spectrum is selected as a pre-burst interval in a duration that is 3-5-times longer than a source time interval depending on the stability of the background. The energy range of the spectral analysis is $15-150 \mathrm{keV}$ for the Swift/BAT data. The energy range to be used in the spectral analysis of the Fermi GBM data is investigated in the following section. The energy response function of Fermi GRB is downloaded from the GBM triggered data $\operatorname{archive}^{2}$ for each GRB. The XSPEC version 12.9.1 software package was used for fitting the spectral data.

\subsection{Identifying the Spectral Energy Range of the Fermi GBM Data}

First, we investigate the energy range of the spectral data of the Fermi GBM by performing the joint spectral fit to the simultaneously detected bright GRBs with the Swift BAT. The Swift BAT has regularly performed the spectral calibration, collecting the Crab nebula data at the specific incident angles [17-19]. Furthermore, the spectral cross-calibration has been performed with the Konus-Wind and the Suzaku/WAM using the simultaneously detected bright GRBs [20]. Therefore, the systematic errors in the energy response function of the Swift BAT are well understood.

The joint spectral analyses of 37 simultaneously detected GRBs by the Fermi GBM and the Swift BAT were conducted. We used the standard energy range between $7 \mathrm{keV}$ and $1 \mathrm{MeV}$ for the Fermi GBM NaI instrument and between $150 \mathrm{keV}$ and $40 \mathrm{MeV}$ for the BGO instrument. Figure 1 shows an example of GRB 170705A. As can be seen in the left panel of Figure 1, there is a noticeable residual from the best fit model at the spectral bins between $7 \mathrm{keV}$ and $30 \mathrm{keV}$ in the Fermi GBM NaI data. The reduced $\chi^{2}$ of the fit is 1.561 in 102 degree of freedom. By ignoring the spectral bins below $30 \mathrm{keV}$ in the Fermi GBM NaI data, the fit was significantly improved with the reduced $\chi^{2}$ of 1.067 in 81 degrees of freedom (right panel of Figure 1). We systematically investigated all 37 GRBs and confirmed that the reasonable joint fit was achieved using above $30 \mathrm{keV}$ for the Fermi GBM NaI data. Therefore, based on this study, we decided to use the energy range between $30 \mathrm{keV}$ and $1 \mathrm{MeV}$ for the Fermi GBM NaI data. The standard energy range between $150 \mathrm{keV}$ and $40 \mathrm{MeV}$ is used for the Fermi GBM BGO data.

\footnotetext{
https:/ / swift.gsfc.nasa.gov/analysis/threads/batspectrumthread.html.

https:/ / heasarc.gsfc.nasa.gov/FTP/fermi/data/gbm/triggers/.
} 

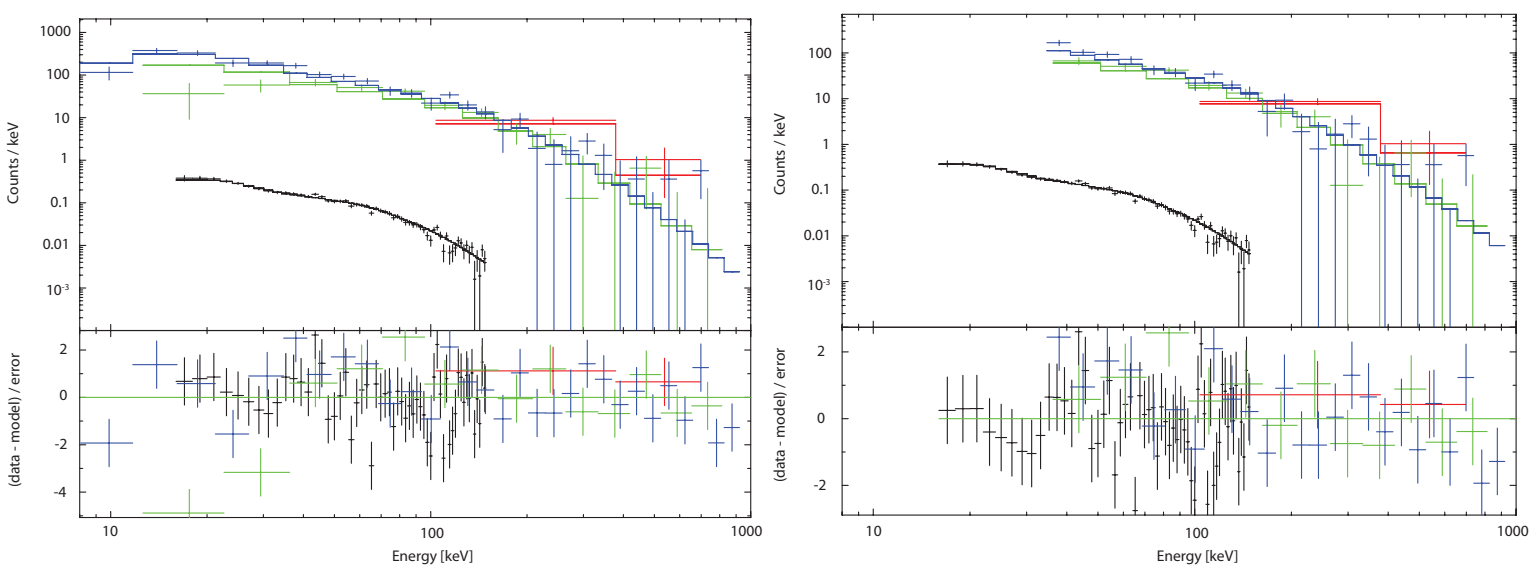

Figure 1. Joint spectral analysis of the Swift BAT (black) and the Fermi GBM NaI (green and blue) and BGO (red) for GRB 170705A (left: using the Fermi GBM NaI data from $7 \mathrm{keV}$ to $1 \mathrm{MeV}$; right: using the Fermi GBM NaI data from $30 \mathrm{keV}$ to $1 \mathrm{MeV}$ ).

\subsection{Preparation of the Data}

We selected 60 short GRBs and 58 long GRBs detected by the Fermi GBM between 2008 and 2017. The selection criteria of long GRBs are that the peak photon flux in $64 \mathrm{~ms}$ reported in the Fermi GBM Burst Catalog [21-23] is $>7.1 \mathrm{ph} \mathrm{cm}^{-2} \mathrm{~s}^{-1}$ and the derived spectral parameters are well constrained. We selected all short GRBs during this period with well constrained spectral parameters. The foreground spectral files are generated using gtbin specifying the $t_{90}$ interval for the time-averaged spectra of short and long GRBs. For the spectrum of the initial $2 \mathrm{~s}$ of long GRBs, we specified the $2 \mathrm{~s}$ window from the trigger time of the Fermi GBM. The background spectral files are generated using gtbin, specifying a pre-burst interval which is $3-5$ times longer than a foreground interval. The data of two triggered $\mathrm{NaI}$ detectors and one BGO detector are used in the analysis. The data and the energy response files are downloaded from the Fermi GBM public data archive available through the Fermi Science Data Center. ${ }^{3}$ The spectral model used in the fit is a cutoff power-law (CPL) model:

$$
f(E)=K_{50}\left(\frac{E}{50 \mathrm{keV}}\right)^{\alpha} \exp \left(\frac{-E(2+\alpha)}{E_{\text {peak }}}\right)
$$

where $\alpha$ is the low energy photon index, $E_{\text {peak }}$ is the peak energy in the $v \mathrm{~F}_{v}$ spectrum and $K_{50}$ is the normalization at $50 \mathrm{keV}$ in units of photons $\mathrm{cm}^{-2} \mathrm{~s}^{-1} \mathrm{keV}^{-1}$.

\section{Results}

Tables 1-3 summarize our GRB samples and derived spectral parameters based on a CPL model fit. Figure 2 shows the distribution of $E_{\text {peak }}$ and $\alpha$ between short GRBs and long GRBs (left panel), and the short GRBs and the initial $2 \mathrm{~s}$ of long GRBs (right panel). The distribution of $\alpha$ for long GRBs tends to overlap at a steeper region (small $\alpha$ ) of short GRBs, whereas $E_{\text {peak }}$ distributes to a higher energy for short GRBs compared to that of long GRBs. On the other hand, the difference in $\alpha$ becomes less evident between short GRBs and the initial $2 \mathrm{~s}$ of long GRBs.

3 https://heasarc.gsfc.nasa.gov/FTP/fermi/data/gbm/bursts/. 
Table 1. Spectral parameters of short GRBs.

\begin{tabular}{|c|c|c|}
\hline GRB & $\alpha$ & $E_{\text {peak }}[\mathrm{keV}]$ \\
\hline GRB081209981 & $-0.48 \pm 0.40$ & $848 \pm 479$ \\
\hline GRB081216531 & $-0.77 \pm 0.18$ & $791 \pm 266$ \\
\hline GRB081223419 & $-1.11 \pm 0.36$ & $301 \pm 127$ \\
\hline GRB081226509 & $0.47 \pm 1.50$ & $269 \pm 133$ \\
\hline GRB090228204 & $-0.49 \pm 0.18$ & $813 \pm 194$ \\
\hline GRB090305052 & $0.01 \pm 0.29$ & $611 \pm 121$ \\
\hline GRB090308734 & $-0.45 \pm 0.22$ & $519 \pm 98$ \\
\hline GRB090617208 & $-0.24 \pm 0.64$ & $823 \pm 623$ \\
\hline GRB090907808 & $0.50 \pm 0.58$ & $339 \pm 59$ \\
\hline GRB091126333 & $-0.39 \pm 2.24$ & $123 \pm 63$ \\
\hline GRB100206563 & $0.10 \pm 0.77$ & $349 \pm 110$ \\
\hline GRB100525744 & $-0.78 \pm 0.81$ & $540 \pm 367$ \\
\hline GRB100625773 & $-0.64 \pm 0.49$ & $504 \pm 251$ \\
\hline GRB100629801 & $-0.94 \pm 0.31$ & $233 \pm 50$ \\
\hline GRB100811108 & $0.31 \pm 0.37$ & $664 \pm 141$ \\
\hline GRB101031625 & $0.34 \pm 1.22$ & $292 \pm 146$ \\
\hline GRB101216721 & $-0.49 \pm 0.25$ & $140 \pm 10$ \\
\hline GRB110526715 & $-0.60 \pm 0.36$ & $422 \pm 146$ \\
\hline GRB110705151 & $-0.06 \pm 0.27$ & $818 \pm 229$ \\
\hline GRB111103948 & $0.35 \pm 0.99$ & $637 \pm 330$ \\
\hline GRB111222619 & $-0.48 \pm 0.29$ & $1147 \pm 550$ \\
\hline GRB120323507 & $-1.40 \pm 0.38$ & $599 \pm 578$ \\
\hline GRB120603439 & $-0.62 \pm 0.50$ & $444 \pm 225$ \\
\hline GRB120811014 & $0.10 \pm 0.39$ & $696 \pm 178$ \\
\hline GRB120817168 & $-0.40 \pm 0.44$ & $763 \pm 448$ \\
\hline GRB120830297 & $0.14 \pm 0.25$ & $737 \pm 132$ \\
\hline GRB121012724 & $-0.30 \pm 0.45$ & $393 \pm 120$ \\
\hline GRB130204484 & $1.52 \pm 3.09$ & $227 \pm 152$ \\
\hline GRB130307126 & $-0.24 \pm 0.82$ & $461 \pm 286$ \\
\hline GRB130628860 & $-0.64 \pm 0.48$ & $561 \pm 331$ \\
\hline GRB130701761 & $-0.56 \pm 0.15$ & $1551 \pm 619$ \\
\hline GRB130912358 & $-0.46 \pm 0.40$ & $409 \pm 129$ \\
\hline GRB131126163 & $1.23 \pm 0.83$ & $474 \pm 92$ \\
\hline GRB131217108 & $0.20 \pm 0.74$ & $697 \pm 495$ \\
\hline GRB140105065 & $-0.79 \pm 0.59$ & $316 \pm 212$ \\
\hline GRB140209313 & $-0.89 \pm 0.08$ & $210 \pm 10$ \\
\hline GRB140511095 & $0.18 \pm 1.98$ & $153 \pm 76$ \\
\hline GRB140605377 & $0.22 \pm 1.00$ & $326 \pm 114$ \\
\hline GRB140626843 & $-1.24 \pm 0.52$ & $108 \pm 21$ \\
\hline GRB140807500 & $-1.01 \pm 0.24$ & $762 \pm 447$ \\
\hline GRB140901821 & $-0.16 \pm 0.33$ & $1033 \pm 420$ \\
\hline GRB141011282 & $-0.32 \pm 0.57$ & $653 \pm 256$ \\
\hline GRB141105406 & $-0.79 \pm 0.33$ & $656 \pm 341$ \\
\hline GRB150118927 & $-0.98 \pm 0.31$ & $551 \pm 323$ \\
\hline GRB150506630 & $-0.44 \pm 0.51$ & $599 \pm 299$ \\
\hline GRB150604434 & $-0.72 \pm 0.51$ & $484 \pm 295$ \\
\hline GRB150811849 & $-0.09 \pm 0.22$ & $899 \pm 205$ \\
\hline GRB150819440 & $-1.28 \pm 0.09$ & $835 \pm 292$ \\
\hline GRB151231568 & $-0.95 \pm 0.30$ & $525 \pm 246$ \\
\hline GRB160406503 & $0.58 \pm 1.22$ & $323 \pm 130$ \\
\hline GRB160804180 & $-0.39 \pm 0.38$ & $555 \pm 245$ \\
\hline GRB160806584 & $-1.44 \pm 0.27$ & $149 \pm 29$ \\
\hline GRB160820496 & $-0.74 \pm 0.56$ & $412 \pm 220$ \\
\hline GRB160821937 & $-0.34 \pm 2.04$ & $101 \pm 37$ \\
\hline GRB160822672 & $-1.06 \pm 0.80$ & $252 \pm 209$ \\
\hline GRB170121133 & $1.82 \pm 4.30$ & $115 \pm 46$ \\
\hline GRB170127634 & $-0.67 \pm 0.73$ & $417 \pm 290$ \\
\hline GRB170206453 & $-0.67 \pm 0.09$ & $418 \pm 36$ \\
\hline GRB170305256 & $-0.54 \pm 0.35$ & $242 \pm 50$ \\
\hline GRB170325331 & $-0.65 \pm 1.21$ & $202 \pm 153$ \\
\hline
\end{tabular}


Table 2. Spectral parameters of long GRBs.

\begin{tabular}{|c|c|c|}
\hline GRB & $\alpha$ & $E_{\text {peak }}[\mathrm{keV}]$ \\
\hline GRB081009140 & $-1.37 \pm 0.14$ & $36 \pm 4$ \\
\hline GRB081215784 & $-0.77 \pm 0.02$ & $566 \pm 17$ \\
\hline GRB090424592 & $-1.16 \pm 0.04$ & $190 \pm 4$ \\
\hline GRB090719063 & $-0.72 \pm 0.04$ & $277 \pm 7$ \\
\hline GRB090804940 & $-0.43 \pm 0.10$ & $103 \pm 1$ \\
\hline GRB091127976 & $-1.98 \pm 0.00$ & $9 \pm 1$ \\
\hline GRB100131730 & $-1.17 \pm 0.08$ & $220 \pm 15$ \\
\hline GRB100324172 & $-0.61 \pm 0.05$ & $483 \pm 25$ \\
\hline GRB100722096 & $-1.88 \pm 0.11$ & $31 \pm 19$ \\
\hline GRB100829876 & $-1.23 \pm 0.08$ & $225 \pm 20$ \\
\hline GRB100910818 & $-1.02 \pm 0.09$ & $179 \pm 10$ \\
\hline GRB101208498 & $-1.44 \pm 0.15$ & $115 \pm 10$ \\
\hline GRB110817191 & $-0.97 \pm 0.07$ & $222 \pm 11$ \\
\hline GRB110921912 & $-1.02 \pm 0.05$ & $643 \pm 76$ \\
\hline GRB111220486 & $-1.09 \pm 0.06$ & $334 \pm 28$ \\
\hline GRB120129580 & $-0.95 \pm 0.03$ & $347 \pm 9$ \\
\hline GRB120204054 & $-1.16 \pm 0.04$ & $198 \pm 6$ \\
\hline GRB120217904 & $-1.19 \pm 0.09$ & $319 \pm 42$ \\
\hline GRB120328268 & $-1.16 \pm 0.03$ & $345 \pm 18$ \\
\hline GRB120426090 & $-0.99 \pm 0.05$ & $148 \pm 2$ \\
\hline GRB120728434 & $-1.41 \pm 0.05$ & $96 \pm 2$ \\
\hline GRB120801920 & $-0.19 \pm 0.50$ & $440 \pm 105$ \\
\hline GRB130121835 & $-1.02 \pm 0.30$ & $235 \pm 50$ \\
\hline GRB130228212 & $-1.53 \pm 0.10$ & $268 \pm 48$ \\
\hline GRB130306991 & $-0.30 \pm 0.84$ & $170 \pm 20$ \\
\hline GRB130425327 & $-1.21 \pm 0.13$ & $252 \pm 27$ \\
\hline GRB130502327 & $-0.92 \pm 0.07$ & $645 \pm 89$ \\
\hline GRB130815660 & $-1.71 \pm 0.15$ & $134 \pm 28$ \\
\hline GRB130821674 & $-1.23 \pm 0.07$ & $493 \pm 80$ \\
\hline GRB131108862 & $-0.90 \pm 0.06$ & $432 \pm 29$ \\
\hline GRB131214705 & $-1.57 \pm 0.07$ & $107 \pm 5$ \\
\hline GRB131229277 & $-0.89 \pm 0.08$ & $360 \pm 30$ \\
\hline GRB140213807 & $-1.70 \pm 0.06$ & $106 \pm 7$ \\
\hline GRB140523129 & $-1.08 \pm 0.03$ & $293 \pm 10$ \\
\hline GRB140621827 & $-0.71 \pm 0.14$ & $571 \pm 119$ \\
\hline GRB140801792 & $-0.33 \pm 0.09$ & $125 \pm 2$ \\
\hline GRB141222298 & $-1.45 \pm 0.07$ & $1275 \pm 796$ \\
\hline GRB150330828 & $-1.10 \pm 0.06$ & $362 \pm 30$ \\
\hline GRB150403913 & $-0.99 \pm 0.03$ & $567 \pm 29$ \\
\hline GRB150426594 & $-0.74 \pm 0.88$ & $112 \pm 24$ \\
\hline GRB151227072 & $-1.06 \pm 0.12$ & $169 \pm 10$ \\
\hline GRB151227218 & $-1.45 \pm 0.05$ & $498 \pm 93$ \\
\hline GRB151231443 & $-0.91 \pm 0.13$ & $209 \pm 12$ \\
\hline GRB160113398 & $0.25 \pm 1.91$ & $140 \pm 45$ \\
\hline GRB160516237 & $-1.60 \pm 0.46$ & $77 \pm 39$ \\
\hline GRB160521385 & $-0.87 \pm 0.05$ & $205 \pm 5$ \\
\hline GRB160724444 & $-1.28 \pm 0.09$ & $200 \pm 18$ \\
\hline GRB16080225 & $-0.79 \pm 0.03$ & $347 \pm 10$ \\
\hline GRB160816730 & $-0.78 \pm 0.04$ & $264 \pm 8$ \\
\hline GRB160910722 & $-0.96 \pm 0.02$ & $457 \pm 14$ \\
\hline GRB161218356 & $-0.69 \pm 0.03$ & $245 \pm 4$ \\
\hline GRB170207906 & $0.00 \pm 0.18$ & $384 \pm 29$ \\
\hline GRB170511249 & $-1.38 \pm 0.10$ & $116 \pm 6$ \\
\hline GRB170522657 & $-0.61 \pm 0.05$ & $387 \pm 15$ \\
\hline GRB170626401 & $-1.26 \pm 0.11$ & $96 \pm 4$ \\
\hline GRB170802638 & $-0.44 \pm 0.65$ & $316 \pm 132$ \\
\hline GRB170826819 & $-0.88 \pm 0.05$ & $422 \pm 24$ \\
\hline GRB171120556 & $-1.23 \pm 0.15$ & $202 \pm 27$ \\
\hline GRB180120207 & $-1.30 \pm 0.04$ & $157 \pm 3$ \\
\hline
\end{tabular}


Table 3. Spectral parameters of the initial $2 \mathrm{~s}$ of long GRBs.

\begin{tabular}{|c|c|c|}
\hline GRB & $\alpha$ & $E_{\text {peak }}[\mathrm{keV}]$ \\
\hline GRB081009140 & $-1.08 \pm 0.25$ & $41 \pm 4$ \\
\hline GRB081215784 & $-0.46 \pm 0.04$ & $699 \pm 32$ \\
\hline GRB090424592 & $-1.02 \pm 0.05$ & $219 \pm 6$ \\
\hline GRB090719063 & $-0.17 \pm 0.09$ & $339 \pm 14$ \\
\hline GRB090804940 & $-0.45 \pm 0.15$ & $114 \pm 3$ \\
\hline GRB091127976 & $-1.67 \pm 0.07$ & $225 \pm 28$ \\
\hline GRB100131730 & $-0.97 \pm 0.08$ & $246 \pm 15$ \\
\hline GRB100324172 & $0.34 \pm 0.09$ & $526 \pm 23$ \\
\hline GRB100722096 & $-1.51 \pm 0.15$ & $65 \pm 7$ \\
\hline GRB100829876 & $-1.00 \pm 0.06$ & $247 \pm 13$ \\
\hline GRB100910818 & $-0.64 \pm 0.87$ & $86 \pm 15$ \\
\hline GRB101208498 & $-1.44 \pm 0.15$ & $116 \pm 10$ \\
\hline GRB110817191 & $-0.54 \pm 0.08$ & $275 \pm 12$ \\
\hline GRB110921912 & $-1.00 \pm 0.09$ & $830 \pm 190$ \\
\hline GRB111220486 & $-0.94 \pm 0.23$ & $428 \pm 149$ \\
\hline GRB120129580 & $-0.95 \pm 0.03$ & $347 \pm 9$ \\
\hline GRB120204054 & $0.50 \pm 0.61$ & $362 \pm 73$ \\
\hline GRB120217904 & $-1.16 \pm 0.09$ & $322 \pm 39$ \\
\hline GRB120328268 & $-1.05 \pm 0.23$ & $615 \pm 360$ \\
\hline GRB120426090 & $-0.87 \pm 0.07$ & $148 \pm 3$ \\
\hline GRB120728434 & $-1.32 \pm 0.24$ & $204 \pm 51$ \\
\hline GRB120801920 & $-0.19 \pm 0.50$ & $440 \pm 105$ \\
\hline GRB130121835 & $-0.52 \pm 0.32$ & $335 \pm 67$ \\
\hline GRB130228212 & $-0.75 \pm 0.34$ & $262 \pm 55$ \\
\hline GRB130306991 & $-0.89 \pm 0.90$ & $572 \pm 506$ \\
\hline GRB130425327 & $-0.83 \pm 0.75$ & $197 \pm 66$ \\
\hline GRB130502327 & $-0.69 \pm 0.66$ & $165 \pm 49$ \\
\hline GRB130815660 & $-1.83 \pm 0.31$ & $162 \pm 149$ \\
\hline GRB130821674 & $-0.56 \pm 0.50$ & $387 \pm 156$ \\
\hline GRB131108862 & $-0.68 \pm 0.09$ & $460 \pm 38$ \\
\hline GRB131214705 & $-0.38 \pm 0.16$ & $405 \pm 53$ \\
\hline GRB131229277 & $-1.13 \pm 0.40$ & $338 \pm 202$ \\
\hline GRB140213807 & $-1.32 \pm 0.08$ & $279 \pm 32$ \\
\hline GRB140523129 & $-0.79 \pm 0.06$ & $458 \pm 31$ \\
\hline GRB140621827 & $-0.80 \pm 0.22$ & $596 \pm 233$ \\
\hline GRB140801792 & $-0.29 \pm 0.16$ & $128 \pm 4$ \\
\hline GRB141222298 & $-1.32 \pm 0.33$ & $144 \pm 35$ \\
\hline GRB150330828 & $0.09 \pm 0.24$ & $358 \pm 39$ \\
\hline GRB150403913 & $-0.84 \pm 0.24$ & $614 \pm 264$ \\
\hline GRB150426594 & $-0.96 \pm 0.75$ & $133 \pm 39$ \\
\hline GRB151227072 & $-0.88 \pm 0.12$ & $181 \pm 10$ \\
\hline GRB151227218 & $-1.15 \pm 0.12$ & $322 \pm 55$ \\
\hline GRB151231443 & $-1.34 \pm 0.20$ & $201 \pm 26$ \\
\hline GRB160113398 & $-0.58 \pm 2.69$ & $96 \pm 55$ \\
\hline GRB160516237 & $-1.50 \pm 0.45$ & $89 \pm 32$ \\
\hline GRB160521385 & $-0.80 \pm 0.06$ & $217 \pm 6$ \\
\hline GRB160724444 & $-1.13 \pm 0.14$ & $475 \pm 136$ \\
\hline GRB16080225 & $-0.49 \pm 0.03$ & $444 \pm 11$ \\
\hline GRB160816730 & $-0.58 \pm 0.07$ & $317 \pm 16$ \\
\hline GRB160910722 & $0.03 \pm 0.80$ & $204 \pm 54$ \\
\hline GRB161218356 & $-1.12 \pm 0.08$ & $277 \pm 22$ \\
\hline GRB170207906 & $1.49 \pm 0.80$ & $155 \pm 13$ \\
\hline GRB170511249 & $0.05 \pm 1.22$ & $96 \pm 17$ \\
\hline GRB170522657 & $-0.33 \pm 0.13$ & $394 \pm 36$ \\
\hline GRB170626401 & $-0.78 \pm 0.13$ & $136 \pm 5$ \\
\hline GRB170802638 & $-0.44 \pm 0.65$ & $316 \pm 132$ \\
\hline GRB170826819 & $-0.47 \pm 0.17$ & $366 \pm 48$ \\
\hline GRB171120556 & $-1.08 \pm 0.06$ & $332 \pm 23$ \\
\hline GRB180120207 & $-0.74 \pm 0.09$ & $210 \pm 10$ \\
\hline
\end{tabular}



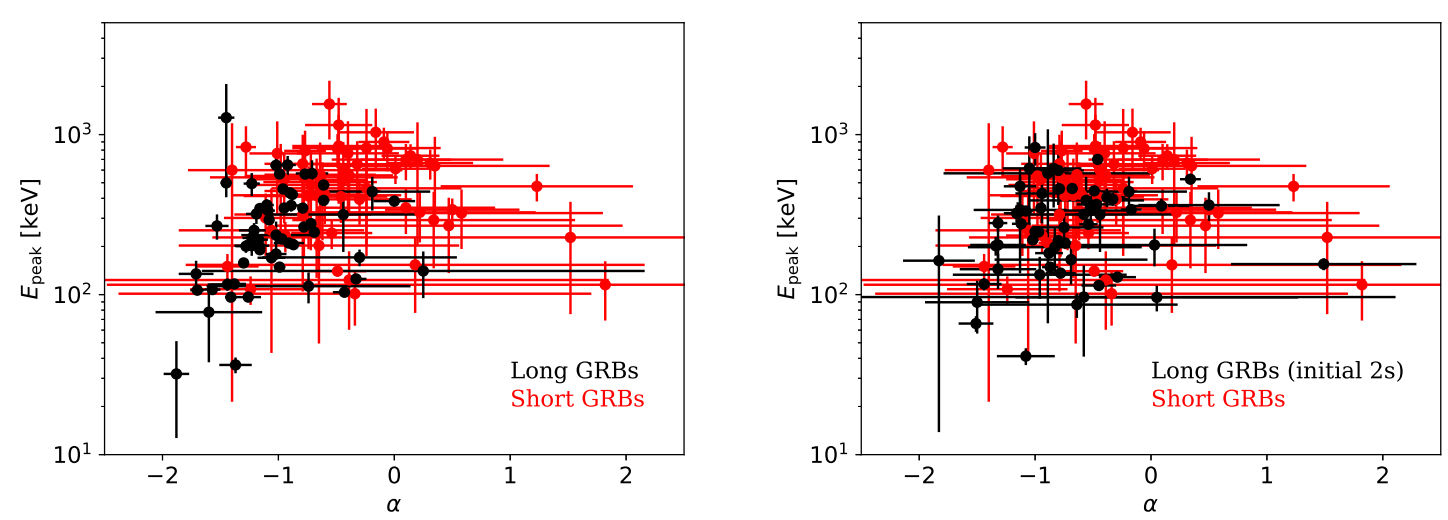

Figure 2. Distribution of $E_{\text {peak }}$ versus $\alpha$ between long GRBs and short GRBs (left), and the initial $2 \mathrm{~s}$ of long GRBs and short GRBs (right).

Figure 3 shows a comparison of the histograms of $E_{\text {peak }}$ between short GRBs and long GRBs (left panel), and short GRBs and the initial $2 \mathrm{~s}$ of long GRBs (right panel). The medians of $E_{\text {peak }}$ are $240.5 \mathrm{keV}, 479.7 \mathrm{keV}$ and $278.6 \mathrm{keV}$ for long GRBs, short GRBs and the initial $2 \mathrm{~s}$ of long GRBs, respectively. The Kolmogorov-Smirnov (K-S) test probabilities of $E_{\text {peak }}$ between short GRBs and long GRBs, and short GRBs and the initial $2 \mathrm{~s}$ of long GRBs are $2.2 \times 10^{-5}$ and $5.3 \times 10^{-5}$, respectively. Therefore, the K-S test shows that the $E_{\text {peak }}$ distributions are drawn from a different population between short GRBs and long GRBs, as well as short GRBs and the initial $2 \mathrm{~s}$ of long GRBs.
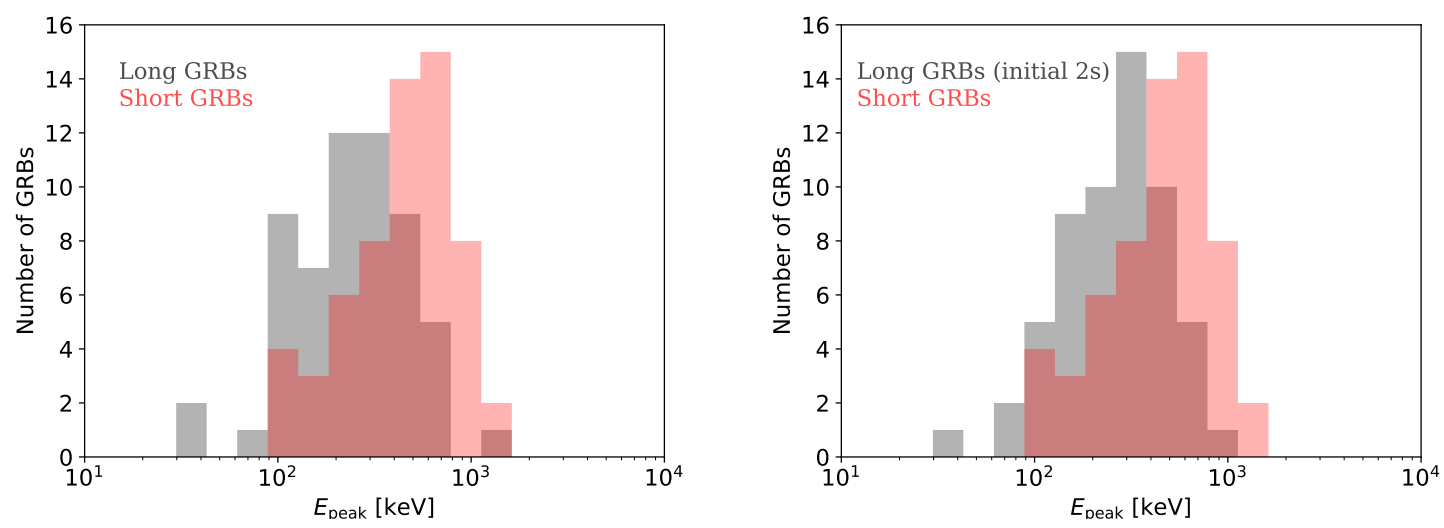

Figure 3. Histograms of $E_{\text {peak }}$ between short GRBs and long GRBs (left), and short GRBs and the initial $2 \mathrm{~s}$ of long GRBs (right).

Figure 4 shows a comparison of the histograms of $\alpha$ between short GRBs and long GRBs (left panel), and short GRBs and the initial $2 \mathrm{~s}$ of long GRBs (right panel). The medians of $\alpha$ are $-1.02,-0.47$ and -0.80 for long GRBs, short GRBs and the initial $2 \mathrm{~s}$ of long GRBs, respectively. The median of $\alpha$ of the initial $2 \mathrm{~s}$ of long GRBs becomes closer to the $\alpha$ distribution of short GRBs. The K-S test probabilities of $\alpha$ between short GRBs and long GRBs, and short GRBs and the initial $2 \mathrm{~s}$ of long GRBs are $1.6 \times 10^{-8}$ and $2.4 \times 10^{-3}$, respectively. This statistical test shows that the $\alpha$ distribution of short GRBs becomes closer to that of the initial $2 \mathrm{~s}$ of long GRBs.

In summary, the spectra of the initial $2 \mathrm{~s}$ of long GRBs show a flatter (harder) $\alpha$ than those of the time-averaged spectra of long GRBs. The distribution of $\alpha$ of the initial $2 \mathrm{~s}$ of long GRBs is closer to that of short GRBs. However, the distribution of $E_{\text {peak }}$ of the initial $2 \mathrm{~s}$ of long GRBs is lower than that of short GRBs, and consistent with the distribution of long GRBs. 

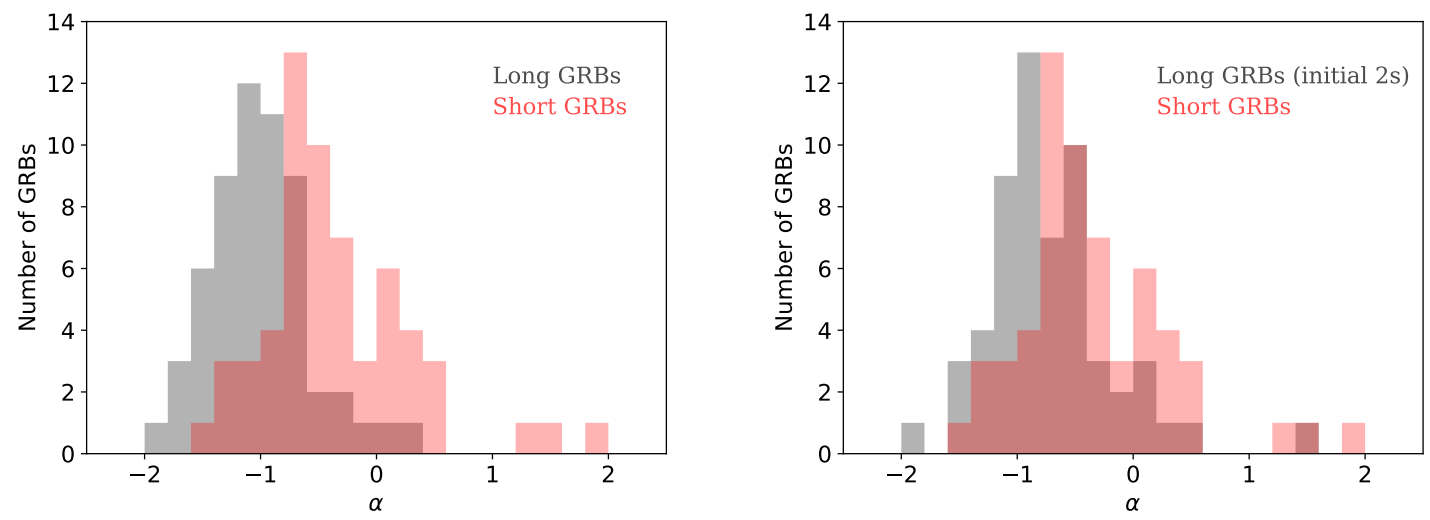

Figure 4. Histograms of $\alpha$ between short GRBs and long GRBs (left), and short GRBs and the initial $2 \mathrm{~s}$ of long GRBs (right).

\section{Discussion}

Ghirlanda et al. [11] investigated the spectral properties of short and long GRBs using BATSE data. They found that short GRBs have a harder $\alpha$ and a higher $E_{\text {peak }}$ compared to those of long GRBs. Furthermore, they also found no difference in $\alpha$ and $E_{\text {peak }}$ between the initial 1-2 s of long GRB and short GRB spectra. Our independent analysis shows a systematically harder $\alpha$ for the initial $2 \mathrm{~s}$ of long GRBs and their $\alpha$ are closer to those of short GRBs. Although the $E_{\text {peak }}$ distributions between short GRBs and the initial $2 \mathrm{~s}$ of long GRBs do not show a statistically significant similarity, the $E_{\text {peak }}$ distribution of the initial $2 \mathrm{~s}$ of long GRBs shows a shift toward a high energy side (Figure 3). Our independent analysis based on the Fermi GBM data confirms the findings by Ghirlanda et al. [11].

The radiation process of a prompt GRB emission is described by synchrotron emission via fast cooling electrons. Therefore, there is a strong restriction on the allowed $\alpha$ from $-3 / 2$ to $-2 / 3$ e.g., [24]. The limitation of a harder side of $\alpha$ is $-2 / 3$. The previous study [25] showed that the spectra of $23 \%$ of the BATSE GRB samples violated the limit. According to our samples, about $9 \%$ of the time-averaged spectra of long GRBs violate the harder side of $\alpha$ by taking into account the error on $\alpha$. On the other hand, $20 \%$ of short GRBs and $24 \%$ of the initial $2 \mathrm{~s}$ of long GRBs violate this limit. Since the similar fractions of the GRB spectra are violating the synchrotron limit for short GRBs and the initial $2 \mathrm{~s}$ of long GRBs, this might indicate that the similar radiation process, which is difficult to achieve by synchrotron emission, is involved in those spectra. Applying the K-S test to the $E_{\text {peak }}$ distributions of the spectra violating and non-violating the synchrotron limit for short GRBs, the initial $2 \mathrm{~s}$ of the long GRBs and long GRBs, we find K-S test probabilities of $7.6 \times 10^{-3}, 8.2 \times 10^{-2}$ and $4.4 \times 10^{-1}$, respectively.

The initial part of a prompt emission spectrum of long GRBs shows a peculiar characteristic in general. A spectral evolution of a prompt emission follows a hard-to-soft trend e.g., [26]. There is a well-known correlation between intensity and hardness during a burst [27]. However, according to Lu et al. [28], the flux and $E_{\text {peak }}$ during a burst do not follow a positive correlation at the initial phase, mainly a rising part of the burst episode. Since a time-averaged spectrum of a long GRB is dominated by the emission from a peak to a tail part of a burst for a single pulse GRB, the distinct spectral characteristic which we see between long GRBs and the initial $2 \mathrm{~s}$ of long GRBs is related to the finding of Lu et al. [28]. A detailed study of the initial part of a GRB spectrum, especially the rising part of a GRB emission, will be important to understand the radiation processes of a prompt GRB emission.

Thanks to the gravitational wave detection accompany with a short GRB, the progenitor of a short GRB has been solved. Therefore, it becomes clear that the progenitors of long and short GRBs are different. Although long and short GRBs are originated to a different progenitor, our results suggest that the similar radiation process is involved between those two different classes of GRBs. We suggest 
that a similar kind of a relativistic jet needs to be launched in both long and short GRBs to explain the similarity in the spectral properties.

Author Contributions: Formal analysis, Y.Y.; Writing—original draft, T.S.; Writing—review and editing, M.S.

Funding: This research received no external funding.

Acknowledgments: We would like to thank the anonymous referees for comments and suggestions that materially improved the paper. This work is supported by MEXT KAKENHI Grant Numbers 17H06357 and 17H06362.

Conflicts of Interest: The authors declare no conflict of interest.

\section{References}

1. Abbott, B.P.; Abbott, R.; Abbott, T.D.; Acernese, F.; Ackley, K.; Adams, C.; Adams, T.; Addesso, P.; Adhikari, R.X.; Adya, V.B.; et al. GW170817: Observation of Gravitational Waves from a Binary Neutron Star Inspiral. Phys. Rev. Lett. 2017, 119, 161101. [CrossRef] [PubMed]

2. Abbott, B.P.; Abbott, R.; Adhikari, R.X.; Ananyeva, A.; Anderson, S.B.; Appert, S.; Arai, K.; Araya, M.C.; Barayoga, J.C.; Barish, B.C.; et al. Multi-messenger Observations of a Binary Neutron Star Merger. Astrophys. J. Lett. 2017, 848, 59. [CrossRef]

3. Goldstein, A.; Veres, P.; Burns, E.; Briggs, M.S.; Hamburg, R.; Kocevski, D.; Wilson-Hodge, C.A.; Preece, R.D.; Poolakkil, S.; Roberts, O.J.; et al. An Ordinary Short Gamma-ray Burst with Extraordinary Implications: Fermi GBM Detection of GRB 170817A. Astrophys. J. Lett. 2017, 848, 14. [CrossRef]

4. Troja, E.; Piro, L.; Van Eerten, H.; Wollaeger, R.T.; Im, M.; Fox, O.D.; Butler, N.R.; Cenko, S.B.; Sakamoto, T.; Fryer, C.L.; et al. The X-ray Counterpart to the Gravitational-wave Event GW170817. Nature 2017, 551, 71-74. [CrossRef]

5. Troja, E.; Piro, L.; Ryan, G.; van Eerten, H.; Ricci, R.; Wieringa, M.H.; Lotti, S.; Sakamoto, T.; Cenko, S.B. The Outflow Structure of GW170817 from Late-time Broad-band Observations. Mon. Not. R. Astron. Soc. Lett. 2018, 478, L18-L23. [CrossRef]

6. Mooley, K.P.; Nakar, E.; Hotokezaka, K.; Hallinan, G.; Corsi, A.; Frail, D.A.; Horesh, A.; Murphy, T.; Lenc, E.; Kaplan, D.L.; et al. A Mildly Relativistic Wide-angle Outflow in the Neutron-star Merger Event GW170817. Nature 2018, 554, 207-210. [CrossRef] [PubMed]

7. Yamazaki, R.; Ioka, K.; Nakamura, T. X-ray Flashes from Off-Axis Gamma-ray Bursts. Astrophys. J. Lett. 2002, 571, L31-L35. [CrossRef]

8. Zhang, B.; Dai, X.; Lloyd-Ronning, N.M.; Mészáros, P. Quasi-universal Gaussian Jets: A Unified Picture for Gamma-ray Bursts and X-ray Flashes. Astrophys. J. Lett. 2004, 601, L119-L122. [CrossRef]

9. Kouveliotou, C.; Meegan, C.A.; Fishman, G.J.; Bhat, N.P.; Briggs, M.S.; Koshut, T.M.; Paciesas, W.S.; Pendleton, G.N. Identification of two classes of gamma-ray bursts. Astrophys. J. 1993, 413, L101-L104. [CrossRef]

10. Ghirlanda, G.; Ghisellini, G.; Celotti, A. The spectra of short Gamma-ray Bursts. Astron. Astrophys. 2004, 422, L55-L58. [CrossRef]

11. Ghirlanda, G.; Nava, L.; Ghisellini, G.; Celotti, A.; Firmani, C. Short versus Long gamma-ray bursts: Spectra, energetics, and luminosities. Astron. Astrophys. 2009, 496, 585-595. [CrossRef]

12. Hakkila, J.; Preece, R.D. Unification of Pulses in Long and Short Gamma-ray Bursts: Evidence from Pulse Properties and their Correlations. Astrophys. J. 2011, 740, 104. [CrossRef]

13. Ghirlanda, G.; Ghisellini, G.; Nava, L. Short and Long Gamma-ray Bursts: Same Emission Mechanism? Mon. Not. R. Astron. Soc. Lett. 2011, 418, L109-L113. [CrossRef]

14. Gehrels, N.; Chincarini, G.; Giommi, P.; Mason, K.O.; Nousek, J.A.; Wells, A.A.; White, N.E.; Barthelmy, S.D.; Burrows, D.N.; Cominsky, L.R.; et al. The Swift Gamma-ray Burst Mission. Astrophys. J. 2004, 611, 1005-1020. [CrossRef]

15. Barthelmy, S.D.; Barbier, L.M.; Cummings, J.R.; Fenimore, E.E.; Gehrels, N.; Hullinger, D.; Krimm, H.A.; Markwardt, C.B.; Palmer, D.M.; Parsons, A.; et al. The Burst Alert Telescope (BAT) on the SWIFT MIDEX Mission. Space Sci. Rev. 2005, 120, 143-164. [CrossRef]

16. Meegan, C.; Lichti, G.; Bhat, P.N.; Bissaldi, E.; Briggs, M.S.; Connaughton, V.; Diehl, R.; Fishman, G.; Greiner, J.; Hoover, A.S.; et al. The Fermi Gamma-ray Burst Monitor. Astrophys. J. 2009, 702, 791-804. [CrossRef] 
17. Lien, A.; Sakamoto, T.; Barthelmy, S.D.; Baumgartner, W.H.; Cannizzo, J.K.; Chen, K.; Collins, N.R.; Cummings, J.R.; Gehrels, N.; Krimm, H.A.; et al. The Third Swift Burst Alert Telescope Gamma-ray Burst Catalog. Astrophys. J. 2016, 829, 47. [CrossRef]

18. Sakamoto, T.; Barthelmy, S.D.; Barbier, L.; Cummings, J.R.; Fenimore, E.E.; Gehrels, N.; Hullinger, D.; Krimm, H.A.; Markwardt, C.B.; Palmer, D.M.; et al. The First Swift BAT Gamma-ray Burst Catalog. Astrophys. J. Suppl. Ser. 2008, 175, 179-190. [CrossRef]

19. Sakamoto, T.; Barthelmy, S.D.; Baumgartner, W.H.; Cummings, J.R.; Fenimore, E.E.; Gehrels, N.; Krimm, H.A.; Markwardt, C.B.; Palmer, D.M.; Parsons, A.M.; et al. The Second Swift Burst Alert Telescope Gamma-ray Burst Catalog. Astrophys. J. Suppl. Ser. 2011, 195, 27. [CrossRef]

20. Sakamoto, T.; Pal'Shin, V.; Yamaoka, K.; Ohno, M.; Sato, G.; Aptekar, R.; Barthelmy, S.D.; Baumgartner, W.H.; Cummings, J.R.; Fenimore, E.E.; et al. Spectral Cross-Calibration of the Konus-Wind, the Suzaku/WAM, and the Swift/BAT Data Using Gamma-ray Bursts. Publ. Astron. Soc. Jpn. 2011, 63, 215-277. [CrossRef]

21. Gruber, D.; Goldstein, A.; von Ahlefeld, V.W.; Bhat, P.N.; Bissaldi, E.; Briggs, M.S.; Byrne, D.; Cleveland, W.H.; Connaughton, V.; Diehl, R.; et al. The Fermi GBM Gamma-ray Burst Spectral Catalog: Four Years of Data. Astrophys. J. Suppl. Ser. 2014, 211, 27. [CrossRef]

22. Von Kienlin, A.; Meegan, C.A.; Paciesas, W.S.; Bhat, P.N.; Bissaldi, E.; Briggs, M.S.; Burgess, J.M.; Byrne, D.; Chaplin, V.; Cleveland, W.; et al. The Second Fermi GBM Gamma-ray Burst Catalog: The First Four Years. Astrophys. J. Suppl. Ser. 2014, 211, 13. [CrossRef]

23. Bhat, P.N.; Meegan, C.A.; von Kienlin, A.; Paciesas, W.S.; Briggs, M.S.; Burgess, J.M.; Burns, E.; Chaplin, V.; Cleveland, W.H.; Collazzi, A.C.; et al. The Third Fermi GBM Gamma-ray Burst Catalog: The First Six Years. Astrophys. J. Suppl. Ser. 2016, 223, 18. [CrossRef]

24. Sari, R.; Piran, T.; Narayan, R. Spectra and Light curves of Gamma-ray Bursts Afterglows. Astrophys. J. Lett. 1998, 497, L17-L20. [CrossRef]

25. Preece, R.D.; Briggs, M.S.; Mallozzi, R.S.; Pendleton, G.N.; Paciesas, W.S.; Band, D.L The Synchrotron Shock Model Confronts a "Line of Death" in the BATSE Gamma-ray Burst Data. Astrophys. J. Lett. 1998, 506, L23-L26. [CrossRef]

26. Norris, J.P.; Share, G.H.; Messina, D.C.; Dennis, B.R.; Desai, U.D.; Cline, T.L.; Matz, S.M.; Chupp, E.L. Spectral Evolution of Pulse Structures in Gamma-ray Burst. Astrophys. J. 1986, 301, 213-219. [CrossRef]

27. Golenetskii, S.V.; Mazets, E.P.; Aptekar, R.L.; Ilinskii, V.N. Correlation between Luminosity and Temperature in Gamma-ray Burst Sources. Nature 1983, 306, 451-453. [CrossRef]

28. Lu, R.J.; Hou, S.J. Liang, E.W. The $E_{\text {peak }}$-Flux Correlation in the Rising and Decaying Phases of Gamma-ray Burst Pulses: Evidence for Viewing Angle Effect? Astrophys. J. 2010, 720, 1146-1154. [CrossRef] 